\title{
RISK VIP: Evaluation of Flood Risk on the French Railway Network Using an Innovative GIS Approach
}

\author{
Mark CHEETHAM ${ }^{1, a}$, François CHIROUZE ${ }^{1}$ and Laurent BREDIER ${ }^{1}$ \\ ${ }^{1}$ SNCF Réseau, Infrastructure, Tracks and Environment Department, La Plaine St Denis, 93574, France
}

\begin{abstract}
Flooding can have significant direct and indirect negative effects on a railway network affecting both infrastructure and rail operations. Such impacts include the delaying or cancelling of train services, damage to railway structures or the implementation of costly maintenance and monitoring programs to ensure the safety and performance of the railway system. Identifying sections of railway line at risk from flooding allows appropriate actions to be targeted at specific areas and contributes to an effective asset management plan. Flooding of railway infrastructure can have numerous sources including surface water run-off, insufficient capacity of hydraulic structures or the inundation of embankments located in floodplains. Consequences of flooding include the destabilisation of structures (surface erosion of embankments or the undermining of bridge foundations), differential settlement of structures and damage to the track structure. This paper details an innovative approach developed at the SNCF using a Geographic Information System (GIS) model to identify zones of the railway network at risk of different types of flooding. The GIS model RiskVIP has been constructed through the assessment of three distinct components of risk: 'Vulnerability' (assessment of the susceptibility of the railway infrastructure to flood conditions), Intensity' (capacity of a catchment to generate a flood flow), Probability' (probability of a rainfall event).Through the application of decision trees, the component 'Intensity' has been characterised in the model by the physical properties of the catchment intercepted by the railway line (surface area of the catchment, slope and land cover characteristics) and 'Vulnerability' by the infrastructure itself (type, geometry and the presence of hydraulic structures). In order to evaluate its efficiency at identifying sites at risk of flooding, the model has been tested in the region of LanguedocRoussillon in France on a reach of over 380km of railway line. In order to characterise the component 'probability', data relating to important historic rainfall events have been used in the model against which known incidents on the railway line have been subsequently analysed. Initial results are very positive with a high level of capture of known incidents by the model in relation to the type of flooding recorded. The model RiskVIP allows the evaluation of flood risk to be undertaken at different scales and will aid in targeting precise reaches of railway line to be studied in more detail. It is a tool which can aid in the management of flood risk on the railway network, optimising for example the maintenance program of drainage structures, ensuring monitoring and inspections are targeted at problem reaches, identifying areas where civil works are necessary and improving the overall resilience of the railway system.
\end{abstract}

\section{Introduction}

Flooding can have significant direct and indirect negative effects on a railway network affecting both rail operations and infrastructure. Such impacts include the delaying or cancelling of train services, causing damage to railway structures or the necessity to implement costly maintenance and monitoring programs to ensure the safety and performance of the system. Identifying sections of railway line at risk from flooding allows appropriate actions to be targeted at specific portions of line and contributes to an effective asset management plan.

The present paper outlines the approach taken to identify and characterize the risks relating to flooding on the rail

\footnotetext{
a Corresponding author: mark.cheetham@sncf.fr
}

network in France. The initial study area covers the region of Languedoc-Roussillon in the south of France (Figure 1). The study is currently at preliminary risk assessment stage.

Due to the large area under consideration (nearly $400 \mathrm{~km}$ of line), it was decided to undertake the evaluation of flood risk using a Geographical Information System (GIS) approach. The following chapters present the way the model was conceived and constructed and show the initial results of the project in terms of identifying earthwork structures in the rail network exposed to flood risk and also how this risk is qualified in terms of type of flooding and the consequences for the railway infrastructure. 


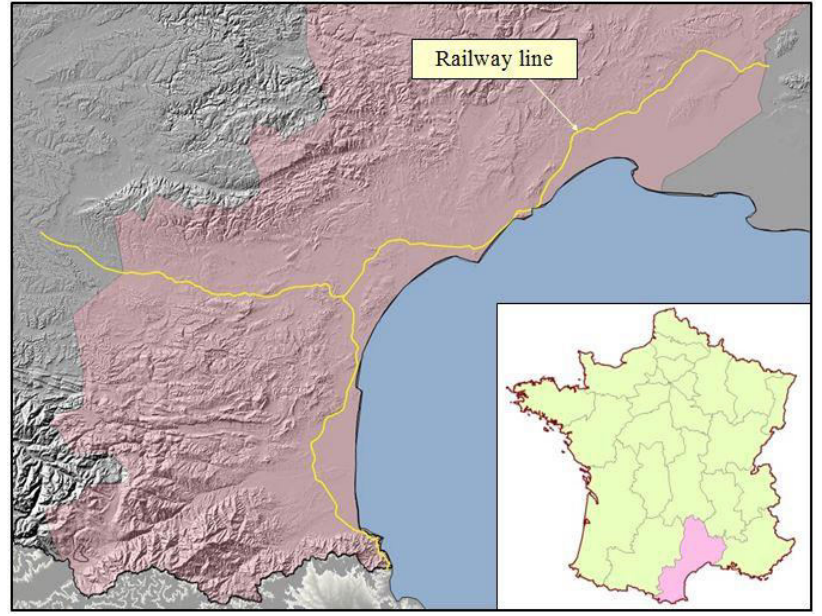

Figure 1- Study Area (Languedoc-Roussillon)

\section{Flood risk management}

\subsection{Flood risk management approach}

The railway network is exposed to many forms of flooding and the management of flood risk is the responsibility of the owner and/ or operator. In all forms of risk management, a procedure for understanding the risks affecting the system being studied is required, which can often require several stages to fully appreciate the sources and consequences of the different types of risk. The diagram presented in Figure 2, proposed in the ISO 31000 document $^{1}$, presents the stages involved in a detailed risk management process from establishing the context of the risk assessment through to proposing a series of options for reducing and monitoring risk.

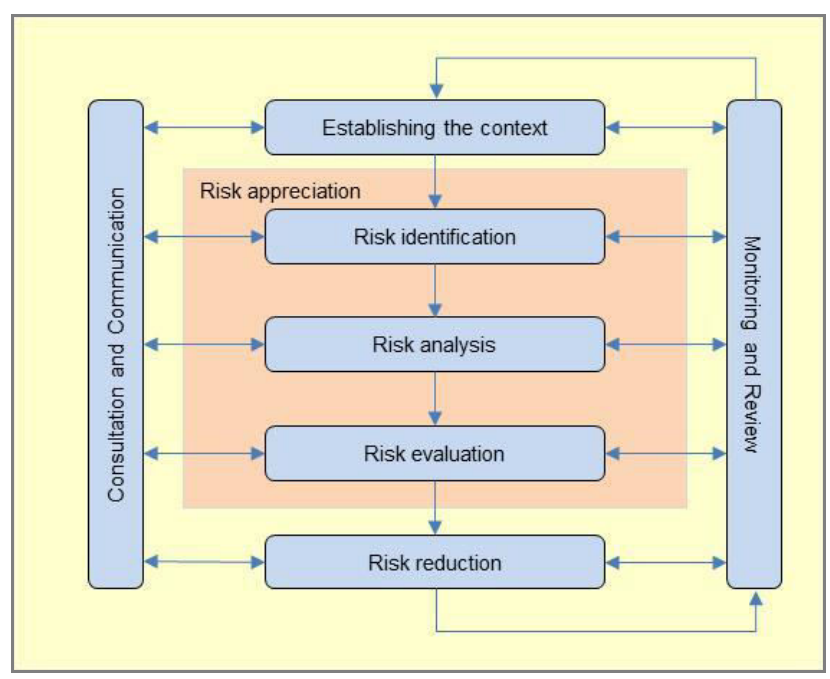

Figure 2 - Risk management process (NF EN 31010)

The following sections detail how the process presented in has been applied throughout this project.

\subsection{Definitions of risk}

Numerous definitions exist for what constitutes a risk and, in establishing the context of a project involving an analysis of risks, it is important to determine what is understood by risk and the components of risk that allow a satisfactory evaluation to be made. ISO Guide 73, Management of risk - Vocabulary ${ }^{\text {Erreur! Source du renvoi }}$ introuvable., defines risk as the "effect of uncertainty on objectives" where an effect is a deviation from the expected (positive or negative) and objectives can have different aspects such as financial, health and safety and environmental goals.

Risk is often expressed in terms of a combination of the likelihood that an event will occur (probability) and a characterization of the consequences of the event:

$$
\text { Risk }=\text { Probability } x \text { Consequence }
$$

where the component "Consequence" can be defined as the outcome of an event affecting objectives and integrates the notions of 'Intensity' of the event and the 'Vulnerability' of the receptor, which in the case presented in this paper, is a railway infrastructure:

\section{Consequence $=$ Vulnerability $\mathrm{x}$ Intensity}

The definition of risk can therefore be expressed through the evaluation of three components (RiskVIP model):

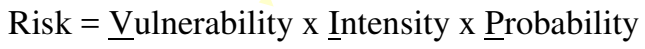

\subsection{Flooding in the context of the railway network}

A railway network is a complex system of different and interacting infrastructure including earthworks, civil structures, track structure, signaling and catenary installations and rail operations (train services, management of stations). For the purposes of the current project, the assessment of risks is limited to earthwork structures although the method described here could be adapted at a later stage to integrate other elements of the system.

Earthworks are present throughout the entire railway network in France $(30000 \mathrm{~km}$ of line) and can be classed into one of three main categories:

- Embankments : where imported material is used in the construction of the line

- Cuttings : where material is excavated during the construction of the line 
- Mixed : a combination of cuttings and embankments

Much of the network in France was constructed in the second half of the $19^{\text {th }}$ Century and is consequently over 100 years old. Railway infrastructure has historically been constructed along valley floors taking advantage of reduced slopes and as such is regularly located in floodplains of watercourses. In addition, due to the linear nature of a railway line, the network often crosses talwegs and other non-permanent or minor watercourses where provision has to be made in the design of the infrastructure for temporary flows arising from surface water runoff from these catchments. As such, the railway network in France is subject to periodic flooding which can have varying degrees of impact ranging from damage to infrastructure or delays for train circulations.

In addition to the problem of flood risk, the network is exposed to a number of other natural hazards such as rockfalls and landslides with an average of 85 incidents per year on the French rail network, of which approximately $25 \%$ are associated with flooding.

\subsection{Risk Identification (Flood risk)}

In the context of the preliminary evaluation of flood risk undertaken in the study area of LanguedocRoussillon, the first stage requires the definition and identification of flood risk sources. A flood can be defined as a "temporary covering of land by water outside its normal confines" 3 . In the characterization of flood risk, the sources of flooding should be clearly identified at the outset to ensure that the consequences of the event can be properly evaluated. As was indicated in the previous section a linear railway system is subject to different types of flooding ranging from long duration but low intensity events to flash floods of very short duration but of high intensity and with often very different consequences. Figure 3 highlights two examples of flood risk to which a railway infrastructure can be exposed.

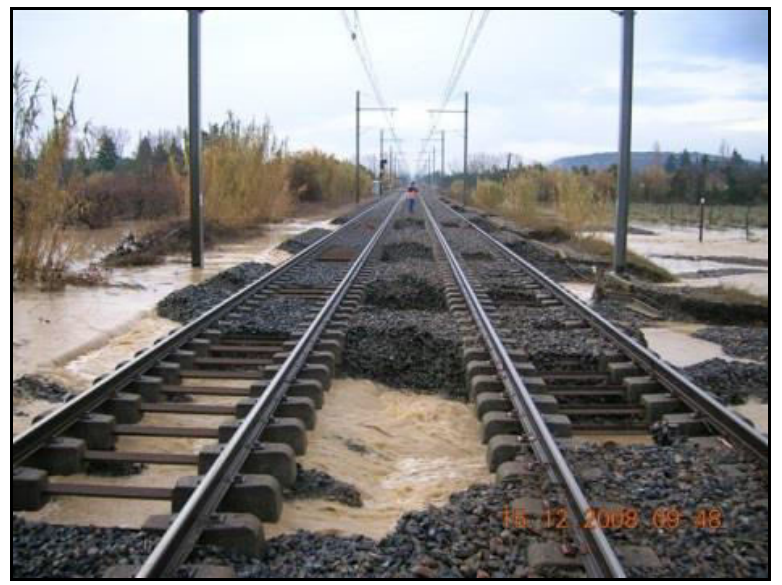

Loss of ballast following the overtopping of an

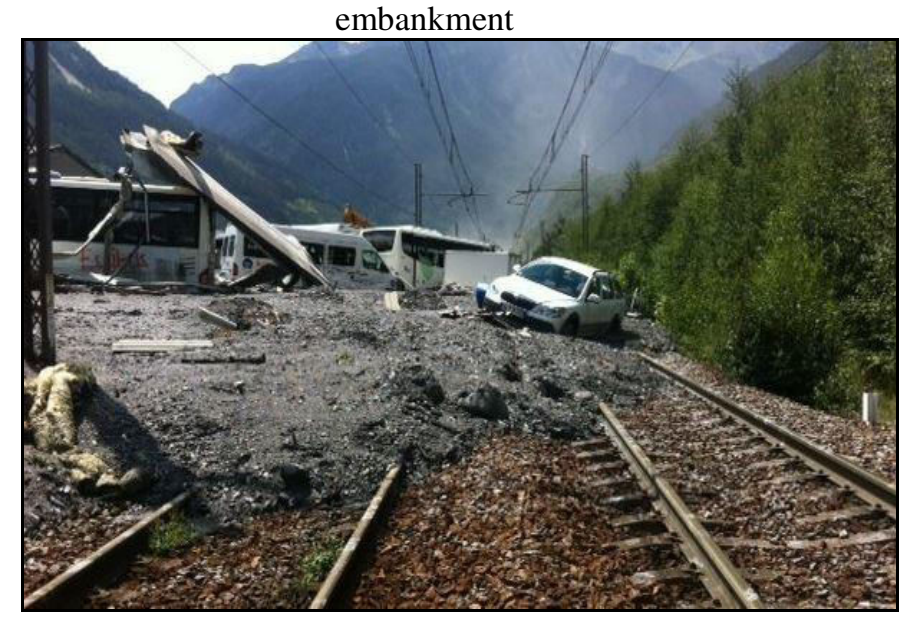

Debris on a railway line following a mudslide Figure 3 - Examples of different types of flood risk on a railway network

The following main sources of flooding were highlighted during the risk identification stage:

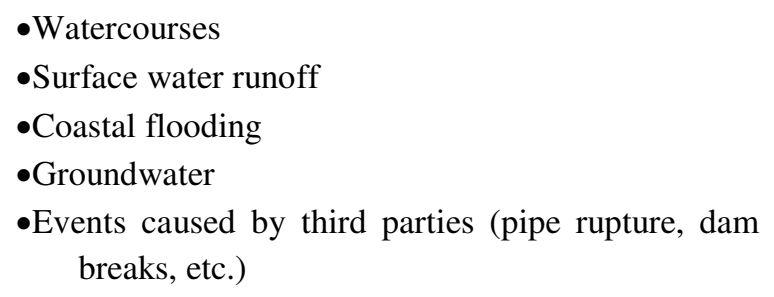

These can be further divided into subgroups to include for the presence of debris in the flow and sediment transport for example.

\subsection{Risk Analysis}

Although the clear definition of the components of risk allows the assessor to subdivide a risk into measurable parameters, it is important to understand and communicate the limits and uncertainty associated with each element. The three components of risk used in our study translate as follows:

- Probability $=$ Return period of the initiating event

- Intensity $=$ Capacity of a catchment to generate a flow

- Vulnerability $=$ Capacity of the railway infrastructure to resist the intercepted flow and thus avoid damage to the structure. In this study, this parameter is predominantly evaluated using the geometrical configuration of the earthwork structure

It should be reiterated that the current paper describes the method undertaken at a preliminary risk assessment 
stage. One of the main objectives of the study is to identify the key parameters which have a role in generating flood risk, avoiding where possible the over complexification of the exercise by, for example, integrating a large number of input criteria to undertake the evaluation. It is envisaged that the results of this preliminary assessment will be used in a more detailed analysis during later stages of the project. Figure 4 presents the three components of risk.

Due to the different types of flood risk under consideration, the large study area $(400 \mathrm{~km}$ of railway line) and the requirement to analyze a large quantity of information, it was decided at an early stage in the project to use of a GIS based model.

It was decided to group the different sources of flood risk identified in Chapter 2.4 into four main categories:
-Catchments for which flood modelling scenarios were readily available (fluvial and maritime)

Flood sources associated with small catchments are typically short duration surface water runoff events and mudslides whereas the larger catchments $\left(<10 \mathrm{~km}^{2}\right)$ will tend to generate longer duration events. This characterization of flood risk in terms of catchment size is the first stage of the flood risk evaluation process and is subsequently refined by integrating other factors in the later stages. The grouping of flood risk sources in terms of catchment size allows different flood sources to be mapped in the GIS model.

Although the model has initially been tested on the region of Languedoc-Roussillon, the intention is that the method should be able to be used for the whole of the railway network in France.

-Catchments of surface area $<1 \mathrm{~km}^{2}$

-Catchments of surface area $1-10 \mathrm{~km}^{2}$

-Catchments of surface area $>10 \mathrm{~km}^{2}$

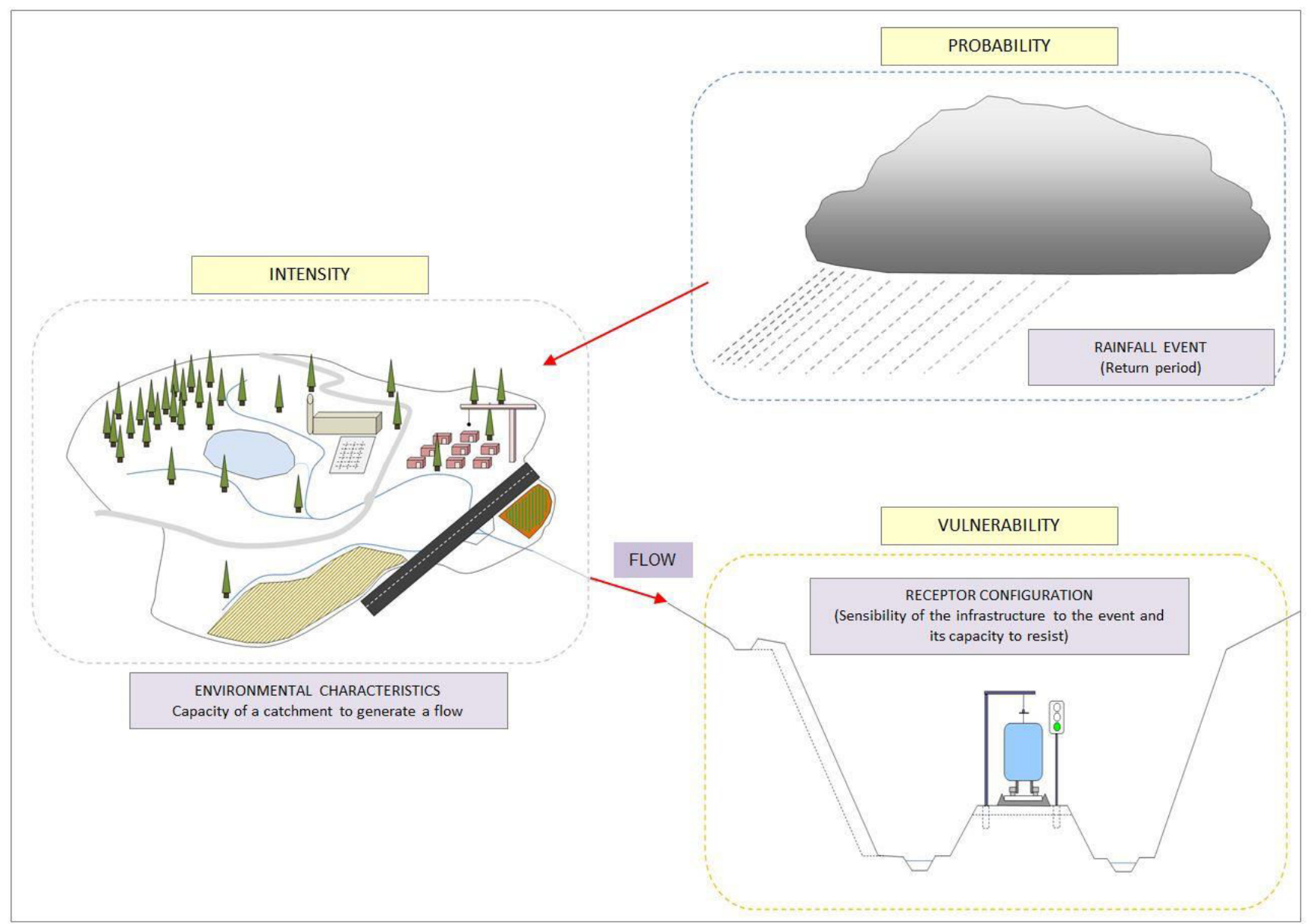

Figure 4 - RISK VIP model 


\subsection{1 'Probability' Component}

The 'probability' component of the model represents the likelihood that the initiating event will occur and is expressed in terms of annual probability of occurrence $(\%)$. The analysis of the probability of an event occurring also takes into account the life expectancy of the infrastructure typically fixed at a duration of 50 years. This results in relatively rare events becoming probable when considered over the lifetime of the structure. For example, the annual probability of the 1 in 100 year flood event is approximately $1 \%$ but considered over the life duration of the structure, the probability of this frequency of event occurring rises to nearly $40 \%$.

It should be noted that the region of LanguedocRoussillon lies in the Mediterranean climatic region and as such experiences very high intensity, low duration rainfall events referred to as Cevennols events, often in the autumn months when air temperatures start to fall and the sea temperature remains relatively high. Rainfall depths of $200 \mathrm{~mm}$ in 24 hours are not uncommon. Due to the very localized nature of these events, it is often very small catchments which are affected. Flows generated by these small catchments can reach $20-50 \mathrm{~m}^{3} / \mathrm{s} / \mathrm{km}^{2}$.

At preliminary risk assessment stage, the evaluation of the probability of an event is based on historical rainfall records or from hydraulic modelling undertaken in the case of more important watercourses.

\subsection{2 'Intensity' Component}

Flood risk mapping has been undertaken on a limited number of watercourses in the LanguedocRoussillon region as part of the French national program to identify flood risk areas (Territoires à Risque d'Inondation, $T R I^{5}$ ). Flood mapping has so far been limited to watercourses where there is a risk to local economies and tends to be associated with rivers flowing through major conurbations. For the watercourses where the maps have been produced, these have been obtained and used in the GIS model as they represent the most detailed information relating to flood risk currently available (Figure 5).

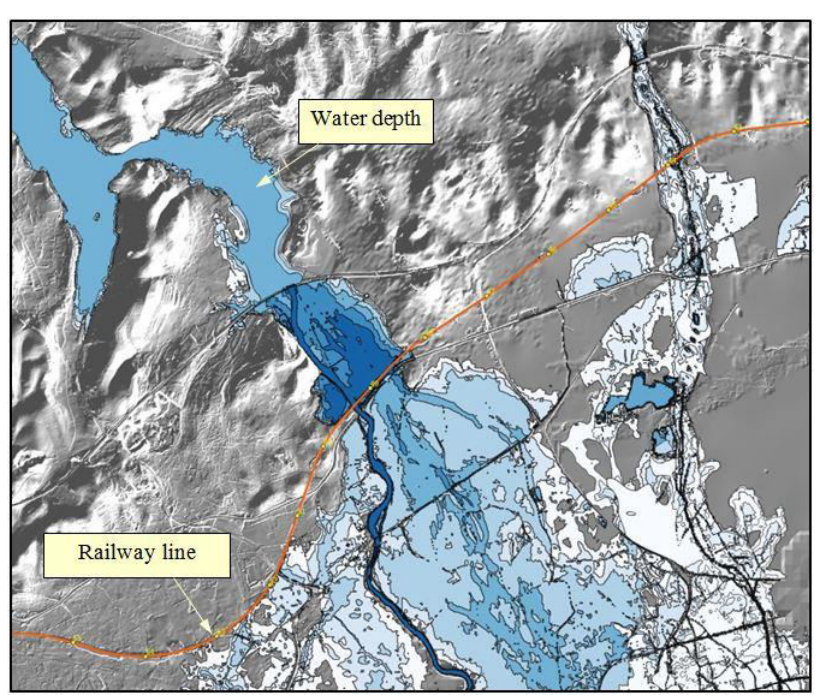

Figure 5 - Example of a TRI flood map showing flood depths

In general terms, maps have been produced for frequent (10-30 year return period), medium (100-300 year return period) and extreme (1000 year) flood events.

Where flood plain maps are not available, which covers most of the catchments intercepted by the railway line in the study area, a different approach was required to identify the sources of flooding and to evaluate the hazard they represent to the railway infrastructure. In order to construct the GIS model, a digital terrain model (DTM) was used with a pixel size of $30 \mathrm{~m}^{2}$. Considering the important length of railway to be analyzed in the study area, this resolution of DTM was deemed sufficient.

For the three catchment sizes detailed previously, decision trees were constructed with a view to characterizing each pixel in the model taking into account two driving parameters:

\section{-Land cover \\ -Topography (Relief)}

The Corine Land Cover database (CLC, 2006 ${ }^{5}$ ) was used to develop the first part of the decision tree. The 44 land cover classes of the CLC were organized into five groups based on the types of land cover and flooding typically experienced in the Languedoc-Roussillon region:

\footnotetext{
-Woodland and garrigue (scrub)

-Prairies

-Non-permanent/ rotational crops

$\bullet$ Vines

-Urban areas
} 
Three slope classes were used in the decision tree to represent in general terms a change in hydraulic regime $(<1 \%, 1-6 \%$ and $>6 \%)$. Within the 'Intensity' decision tree, a qualitative approach was adopted for evaluating the level of intensity corresponding to each class of land cover and topography value. Each pixel in the DMT was subsequently classed as having an intensity value of 'Low', 'Medium' or 'High' intensity. The next stage required identifying each catchment intercepted by the railway line, calculating its size followed by undertaking a qualitative interpretation of its intensity (Figure 6).
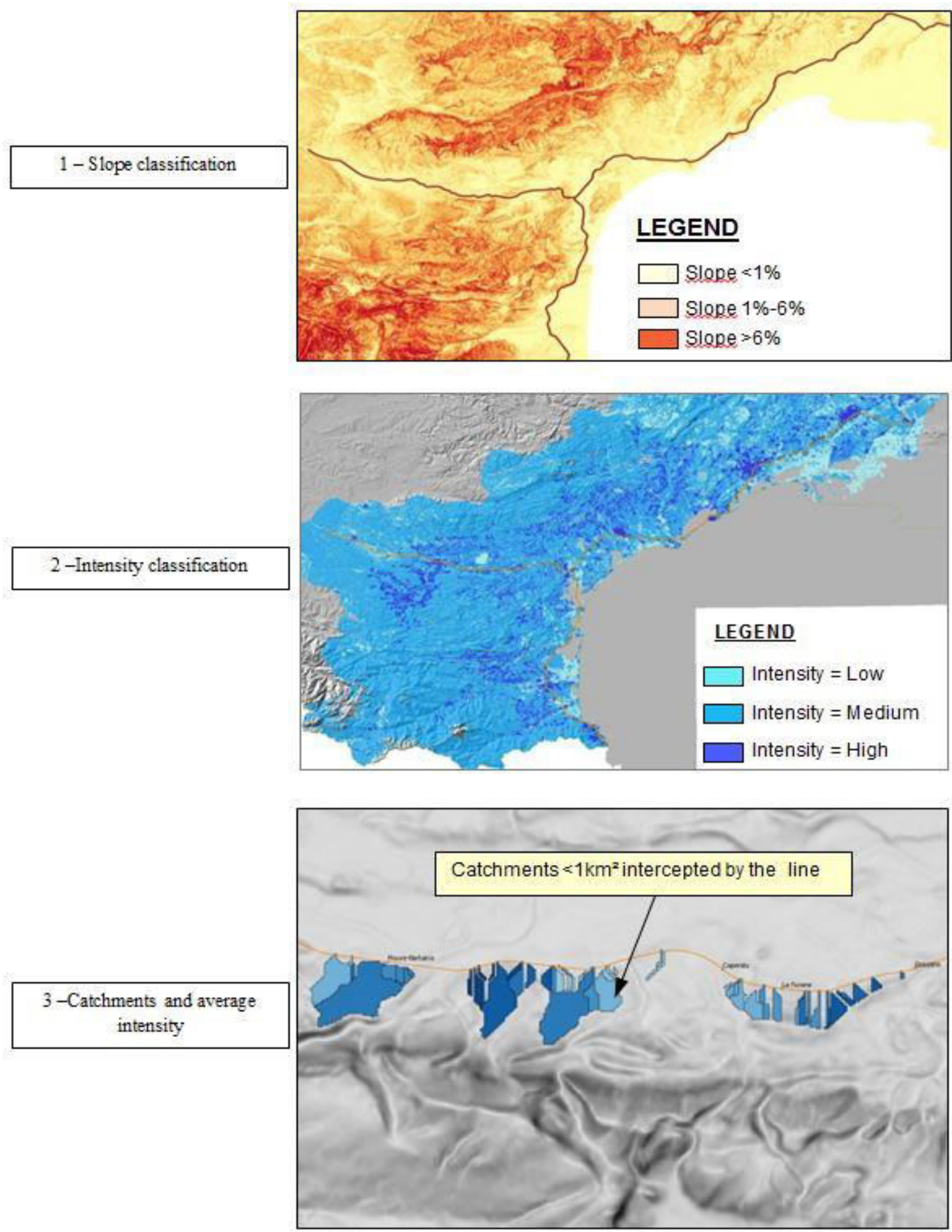

Figure 6 - Process of classifying the « Intensity » parameter for each catchment 
The last stage (3) was repeated for the three catchment sizes described previously.

\subsection{3 'Vulnerability' Component}

The 'Vulnerability' component is possibly the most complex of the three elements of the risk analysis to evaluate, requiring a comprehensive understanding of railway earth structures, their level of exposure to flooding and their functioning within the railway system as a whole. Numerous factors influence the sensibility of an earth structure to flooding including its geometric characteristics (height, base and crest width and embankment slopes for example), the material used in its construction and any flood protection measures that are in place.

As was the case for the component 'Intensity', a decision tree was constructed to evaluate the level of vulnerability of the earth structures in the study area. The three main parameters included in the decision tree include type of earth structure, its geometry and determining whether a hydraulic structure is present at the point where the railway intercepts the catchment.

\subsubsection{Preliminary Results}

Using historical known flooding incidents on the railway line in the region of Languedoc-Roussillon (143 total), the GIS model was tested to evaluate its efficiency at identifying areas at risk from flash flooding associated with sediment transport and mudflows. These types of events are typically associated with small catchments draining steep slopes, two parameters included in the 'Intensity' decision tree.

Of the twenty four incidents of this type registered in the study area, twenty one were correctly located at the intersection point of a catchment basin with an area less than $1 \mathrm{~km}^{2}$ and over half were associated with a "High" average catchment intensity measure. Considering all of the sites with a known history of incidents in the region, $74 \%$ were evaluated as being "Vulnerable" and $24 \%$ as "Moderately vulnerable" with only 2\% scoring a "Low vulnerability". These two tests provide encouraging results for the validation of the approach in evaluating the components 'Intensity' and 'Vulnerability'.

\subsection{Risk Evaluation}

As has been previously indicated, evaluation of the level of flood risk on the network in the region of Languedoc-Roussillon requires the combination of the three components 'Vulnerability', 'Intensity' and 'Probability'. At this preliminary risk assessment stage, considering flood risk at a regional level, the model allows the sites most sensitive to flooding to be identified (i.e. where the consequences of flooding are likely to be the most significant). A matrix for prioritizing the most sensitive sites to be studied in more detail at during the later stages of the project is proposed in Figure 7 (P1 having the highest priority, $\mathrm{P} 4$ the lowest).

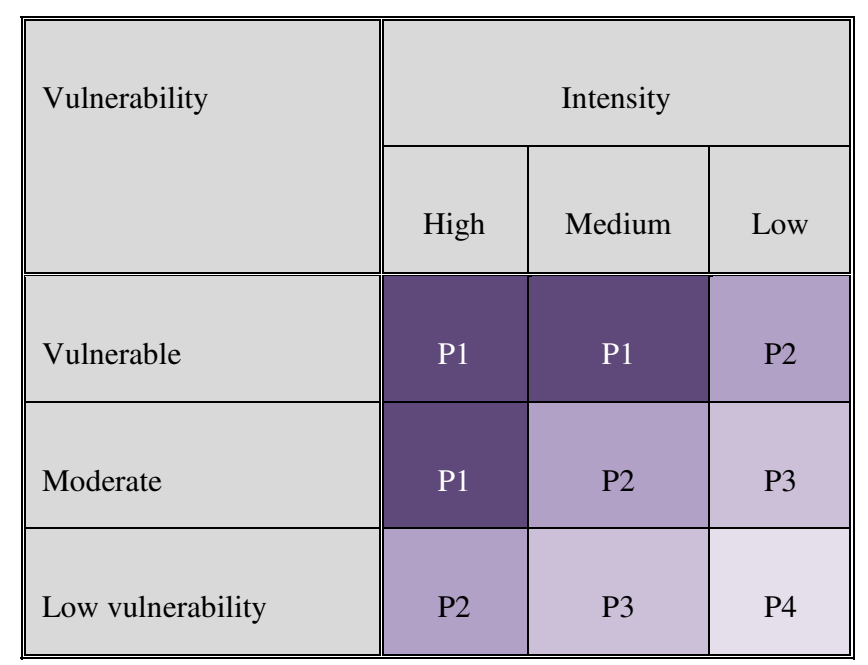

Figure 7 - Priorisation matrix based on the 'Intensity' and 'Vulnerability' components (Consequences)

A test of the efficiency of this matrix to identify sensitive sites to flooding has been undertaken by comparing the total number of historical incidents in the region in terms of the analysis derived from the components 'Intensity' and 'Vulnerability' estimated from the model. As can be seen from Figure 8, nearly $60 \%$ of historical incidents occurred in sections of line evaluated as Priority 1 (highest priority) and over $85 \%$ fall in the Priority 1 and 2 categories. 


\begin{tabular}{|c|c|c|c|c|c|}
\hline \multirow[t]{2}{*}{ Vulnerability } & \multicolumn{3}{|c|}{ Intensity } & \multirow{2}{*}{$\begin{array}{l}\text { Not captured } \\
\text { by the model }\end{array}$} & \multirow[t]{2}{*}{ Total } \\
\hline & High & Medium & Low & & \\
\hline Vulnerable & 29 & $\overline{44}$ & - & \multirow[b]{4}{*}{11} & $\overline{73}$ \\
\hline Moderate & 12 & 38 & - & & 50 \\
\hline Low vulnerability & - & 9 & - & & 9 \\
\hline Not captured & & & & & 11 \\
\hline Total & 41 & 91 & - & 11 & 143 \\
\hline
\end{tabular}

Figure 8 - Evaluation of the consequences of flooding

A number of historical rainfall events that resulted in incidents on the railway line were studied to better understand the component 'Probability' and its relation to the other two components of risk described previously. An example is the event of 11 April 2002 (Figure 9), which although not being exceptional in terms of rainfall depths for the region of Languedoc-Roussillon (150mm in 24 hours), resulted in a number of flooding incidents on the railway line. A spatial analysis of the event indicates that, on a relatively homogenous portion of line, certain sites were at higher risk than others having received less rainfall, all other parameters within the model being equivalent.

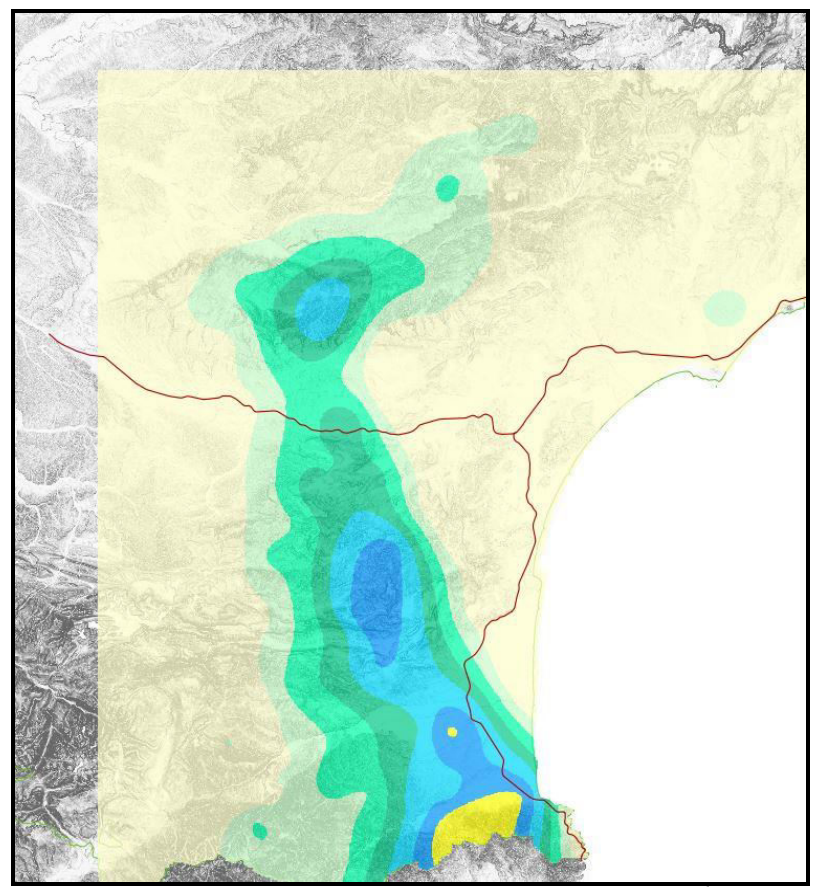

Figure 9 - Rainfall event of 11 april $2002^{6}$

The component 'Probability' is currently under development within the model and should be better characterized during the next stage of the project. Developments to the probability component include a more detailed analysis of return period estimation at a local scale $\left(1 \mathrm{~km}^{2}\right.$ using the method developed by IRSTEA, SHYREG) and undertaking a spatial analysis of historic rainfall events. This will lead to an improved characterization of flood risk to earthwork structures in the study area and on the rail network as a whole.

\subsection{Risk Reduction}

The final stage of the risk management process is the identification of risk reduction measures. Due to the project currently being at preliminary risk assessment stage, it is premature to commence proposing remediation measures without a more detailed risk assessment undertaken at a more local scale. This is the objective of the next stage of the project.

\section{Perspectives}

Preliminary results of the RiskVIP method are promising and appear to show that the model accurately locates earthwork structures which are sensitive to flooding and characterize the consequences of flooding events. A high proportion of historical flood events and incidents are precisely located by the model and the gravity of the consequences is properly characterized.

As has been previously indicated, there remain some improvements to be made to the component 'Probability' which are to be undertaken in the next stage of the project. Improvements are also envisaged in terms of quality of the data in the model, notably the resolution of the DTM which will be reduced to $5 \mathrm{~m}^{2}$ during the next stage. It is expected that this will improve the mapping of drainage paths and the component 'Intensity'.

The model will be used in the next phase of the project to develop the work already undertaken to evaluate how the impact of changes in the catchments intercepted by the railway network have impacted the overall level of flood risk on the railway network. Measuring the impact of changes in catchment characteristics will have an important direct impact on the component 'Intensity' which can subsequently be converted into the component 'Probability'. As has been previously indicated, much of the railway network in France was constructed during the second half of the $19^{\text {th }}$ Century and so is already over 100 years old. Since the original design and construction of the network, significant changes to certain catchments 
has been experienced, particularly due to urban expansion but also due to changes in agricultural practices. Expansion of urban areas tends to reduce the infiltration capacity of natural catchments which can lead to increased rates of surface water runoff, shorter time to peak durations and higher peak flows if no mitigation measures are adopted. Assuming there have been no changes to the railway infrastructure to accommodate these changes, it can be assumed that, for an equivalent rainfall event, the probability of exceedance of the capacity of hydraulic structures will be greater in those catchments where significant changes have taken place. Using historical data from the CLC database, the model is used to initially identify where changes have taken place in the region and then evaluate the impact of these changes. The CLC database has three years of evaluation, 1990, 2000 and 2006. As can be seen from Figure 10, the model is used initially to detect important changes in land use between 1990 and 2006 and then evaluate its impact (positive or negative) in terms of runoff generation. In the example provided, a catchment has been heavily modified through the construction of houses on agricultural land, a comparison of aerial photographs taken from 1990 and 2006 confirm the changes.

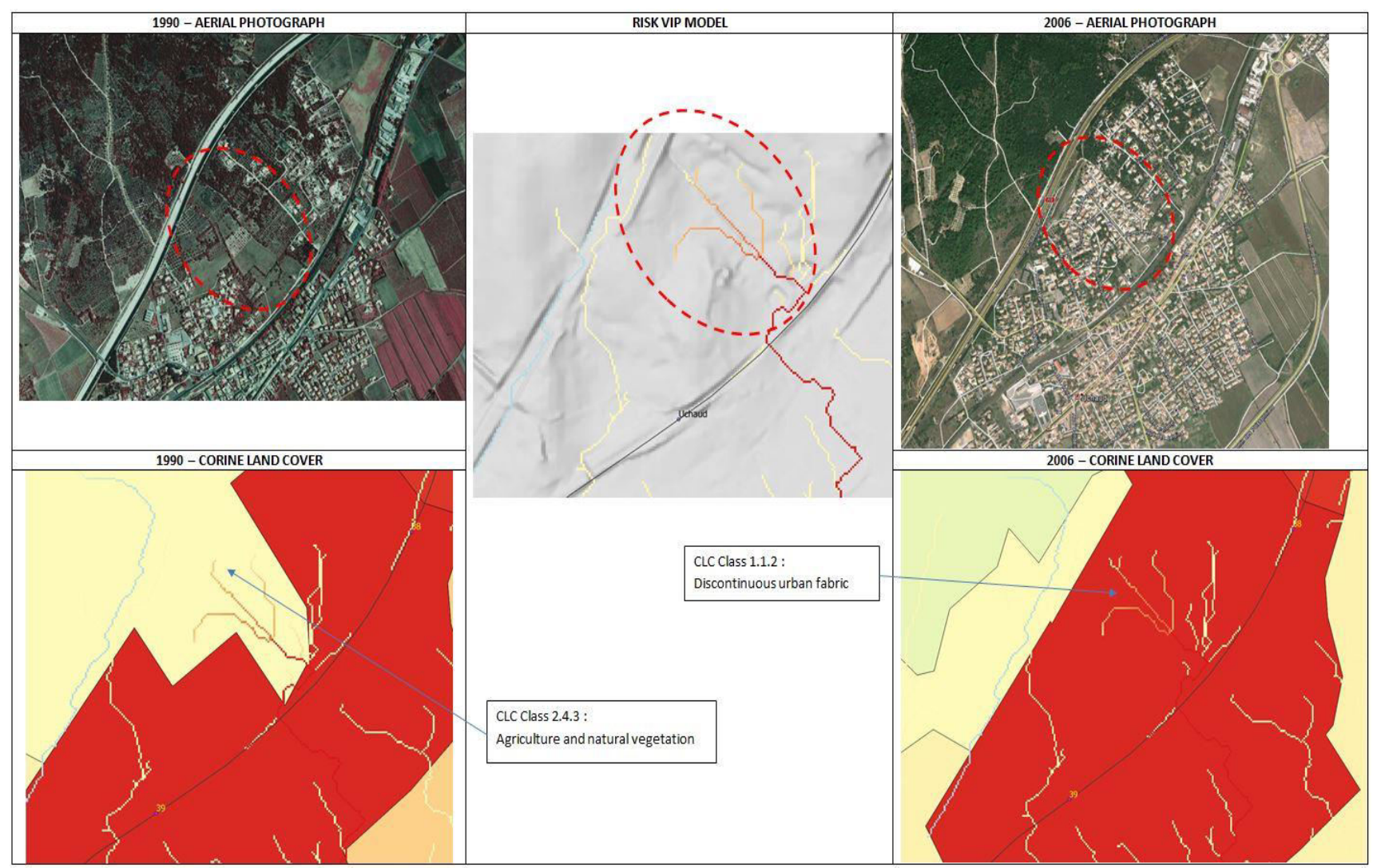

Figure 10 - Example of detection by the model of changes in catchment characteristics and evaluation of their impact.

Through the construction of the Risk VIP model, it should also be possible to evaluate how climate change could affect the risk of flooding to the railway infrastructure in the future.

\section{Conclusions}

This paper has presented an innovative approach to flood risk assessment through the use of a GIS model in the context of earthwork structures on a rail network, which can subsequently be used in the overall system of flood risk management in the next stages of the project. The model is adapted for large scale flood risk analysis, at a national or regional scale and identifies key sections of the network susceptible to be at risk from flooding.
The use of existing hydraulic modelling results where these exist, decision trees to identify catchments with the potential for generating high, medium and low intensity flood events and a comprehensive understanding of railway infrastructure to undertake the vulnerability assessment allows the railway infrastructure manager to prioritize sites to be studied in more detail in the later phases of the project. Historical records of incidents relating to flooding on the rail network in the region of Languedoc-Roussillon originating from flooding confirm the presence of flood risk on a high percentage of sites identified as at risk by the model. In addition, the approach of flood risk based on catchment size aids in the characterization of the consequences of flood risk including long duration low intensity floods and more rapid high intensity flood events. 


\section{References}

1. ISO31000, January 2010, Risk management Principles and guidelines

2. FD ISO Guide 73, December 2009, Management $d u$ risque, Vocabulaire

3. FLOODsite report No.T32-04-01, March 2005, Language of Risk - Project Definitions

4. www.developpement -durable.gouv.fr (Ministere de l'Ecologie, du Developpement Durable et de l'Energie, MEDDE)

5. www.statistiques.developpement-durable.gouv.fr (Ministere de l'Ecologie, du Developpement Durable et de l'Energie, MEDDE)

6. www.pluiesextremes.meteo.fr 